\title{
Blooming flowers from drying drops
}

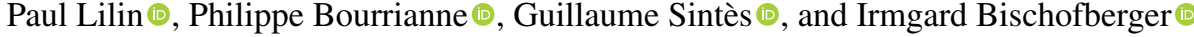 \\ Department of Mechanical Engineering, Massachusetts Institute of Technology, \\ Cambridge, Massachusetts 02139, USA
}

(Received 7 August 2020; published 12 November 2020)

\begin{abstract}
This paper is associated with a video winner of a 2019 American Physical Society's Division of Fluid Dynamics (DFD) Milton van Dyke Award for work presented at the DFD Gallery of Fluid Motion. The original video is available online at the Gallery of Fluid Motion, https://doi.org/10.1103/APS.DFD.2019.GFM.V0082.
\end{abstract}

DOI: 10.1103/PhysRevFluids.5.110511

Thin layers of mud or paint often crack when they dry. Similarly, a drop of a concentrated colloidal suspension left to dry will eventually end up as a fractured solid deposit of particles. Between the drop deposition and the final failure, however, the drop undergoes a sequence of remarkable morphological changes [1]. It separates into different phases and then cracks and bends, exposing a hidden elegance behind the mechanism of failure.

We deposit a millimetric drop of an aqueous suspension of silica nanoparticles (Ludox AS-40, $22 \mathrm{~nm}$ in diameter) on a horizontal glass slide. The drop wets the hydrophilic slide and adopts a contact angle of $22^{\circ}$. As the water evaporates, the particles accumulate at the contact line and build up a thin deposit of close-packed particles. This deposit grows, while the liquid recedes towards the center, as seen in Fig. 1. Stresses build up in the deposit and are suddenly released by the formation of a radial crack. From that first crack, regularly spaced cracks propagate with avalanchelike dynamics over the deposit, creating petals of a colloidal flower. The petals continue to grow towards the center of the drop and bend upward: The flower blooms.

To quantify the crack propagation and the bending of the petals, we image the drop from below with an interferometry technique using monochromatic light of wavelength $\lambda=532 \mathrm{~nm}$. The black and white fringes denote changes in the air-layer thickness beneath the deposit as it detaches from the glass slide; a transition from black to white corresponds to a thickness change of $\lambda / 4=133 \mathrm{~nm}$. We observe bending in two directions. Upon the formation of a crack, the deposit delaminates from the substrate and bends sideways away from the crack, as shown in the top images in Fig. 2. This process induces the propagation of the next crack and gives rise to the avalanchelike behavior. Once a petal is created, it starts to bend radially, increasing its curvature as it grows, as evidenced by the closer spacing of the interference fringes (Fig. 2, bottom images).

The initial particle volume fraction sets the thickness of the deposit, which subsequently governs both the curvature during bending and the width of the petals. The crack spacing increases with film thickness [2]; thus drops of larger particle volume fraction form fewer cracks. Furthermore, the evaporation-induced stresses are larger at the top than at the bottom of the deposit, which leads to a bending moment acting on the petal [3]. Since the bending stiffness increases as the thickness

Published by the American Physical Society under the terms of the Creative Commons Attribution 4.0 International license. Further distribution of this work must maintain attribution to the author(s) and the published article's title, journal citation, and DOI. 

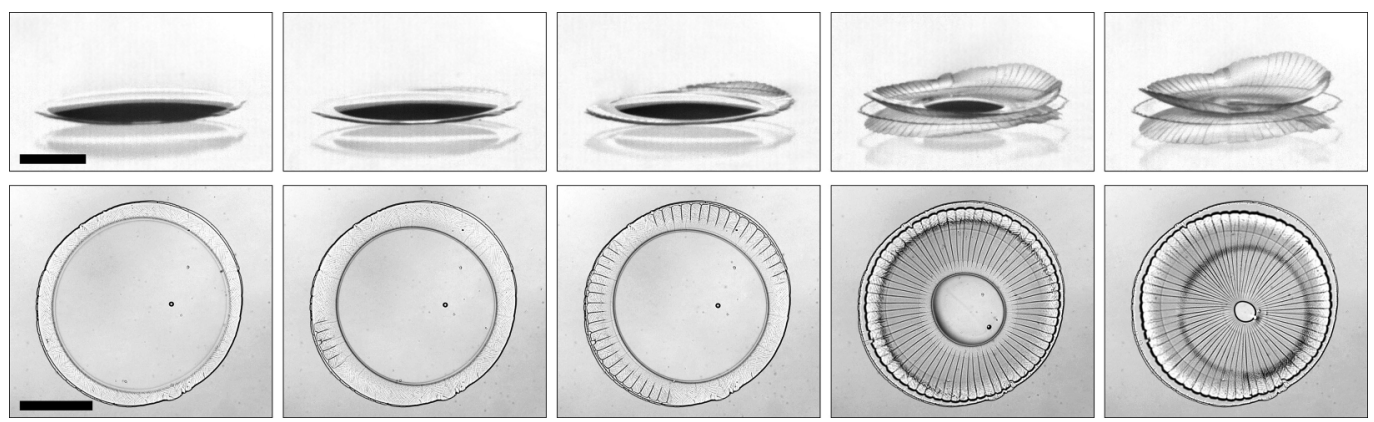

FIG. 1. Drying process of a $0.3-\mu \mathrm{l}$ drop of an aqueous nanoparticle suspension at a particle volume fraction $\phi=7 \%$. As the liquid recedes towards the center, a thin particle deposit builds up from the drop edge, as seen from the side (top row) and the bottom (bottom row). The scale bars represent $1 \mathrm{~mm}$. https://doi.org/10.1103/ APS.DFD.2019.GFM.V0082.
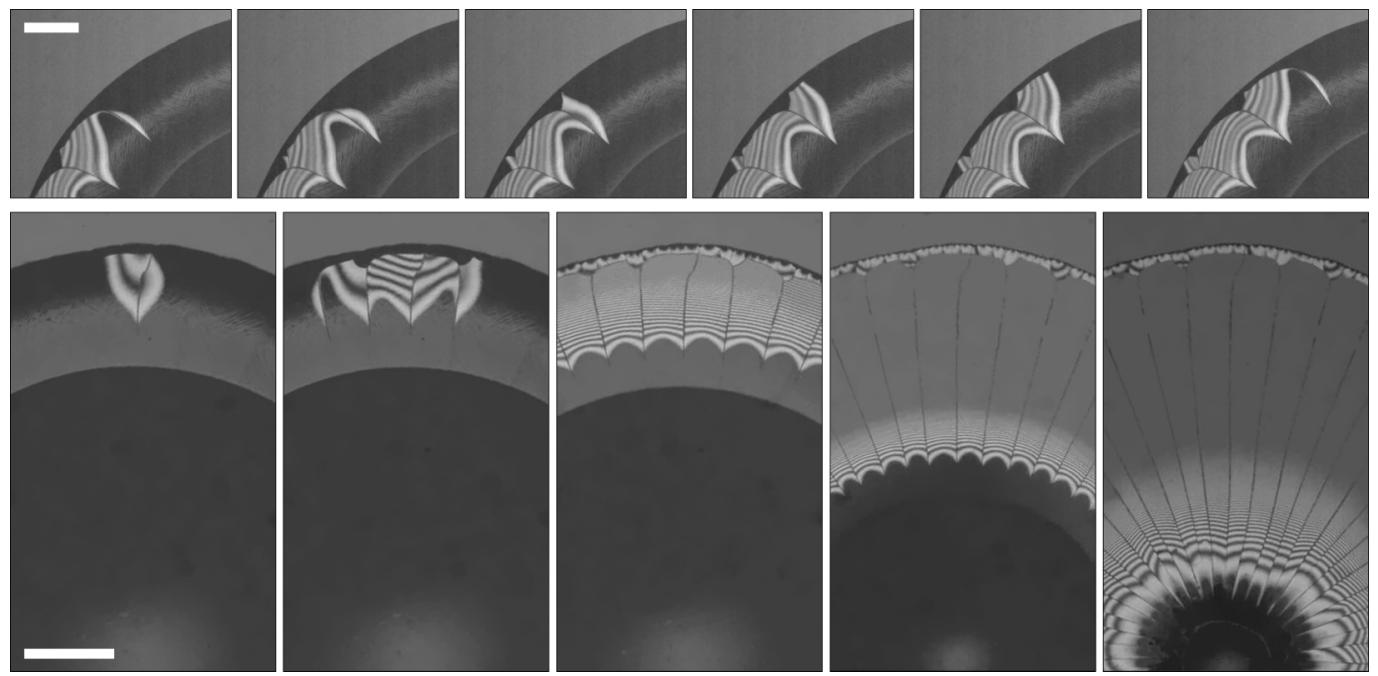

FIG. 2. Microscopy interference images of the petal delamination and bending. The top row shows that after cracking, the deposit delaminates from the substrate and bends sideways, which induces an avalanchelike crack propagation. The time interval between the images is $44 \mathrm{~ms}$. The bottom row shows that as the petals grow, they bend radially. The total time is $42 \mathrm{~s}$. The scale bars represent $200 \mu \mathrm{m}$. https://doi.org/10.1103/APS. DFD.2019.GFM.V0082.

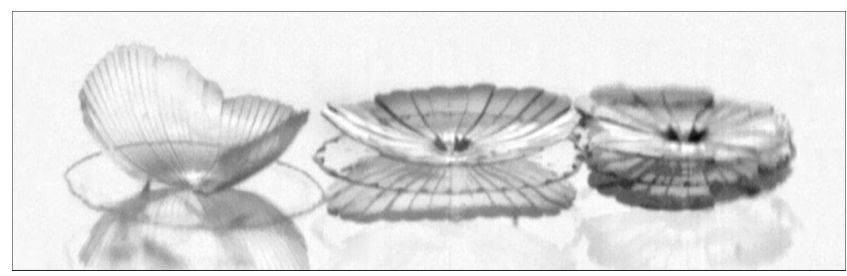

FIG. 3. Blooming flowers created by drying suspension drops of volume fractions $\phi=7 \%, 16 \%$, and $20 \%$ (from left to right). The width of the image is $8.6 \mathrm{~mm}$. https://doi.org/10.1103/APS.DFD.2019.GFM.V0082. 
cubed, thicker petals bend less. This leads to a stunning variety of flower shapes for different particle volume fractions, as shown in Fig. 3 for drops of $\phi=7 \%-20 \%$. The colloidal flowers are transient testimonies of the remarkable complexity of drying phenomena.

[1] F. Parisse and C. Allain, Opening of a glass flower, Phys. Fluids 8, S6 (1996).

[2] C. Allain and L. Limat, Regular Patterns of Cracks Formed by Directional Drying of a Colloidal Suspension, Phys. Rev. Lett. 74, 2981 (1995).

[3] F. Giorgiutti-Dauphiné and L. Pauchard, Dynamic delamination of drying colloidal films: Warping and creep behavior, Colloid. Surf. A 466, 203 (2015). 